\title{
The MEarth project: searching for transiting habitable super-Earths around nearby M dwarfs
}

\author{
Jonathan Irwin, David Charbonneau, Philip Nutzman, \\ and Emilio Falco
}

Harvard-Smithsonian Center for Astrophysics,

60 Garden Street, Cambridge, MA 02138, USA

email: jirwin@cfa.harvard.edu

\begin{abstract}
Due to their small radii, $M$ dwarfs are very promising targets to search for transiting super-Earths, with a planet of 2 Earth radii orbiting an M5 dwarf in the habitable zone giving rise to a $0.5 \%$ photometric signal, with a period of two weeks. This can be detected from the ground using modest-aperture telescopes by targeting samples of nearby M dwarfs. Such planets would be very amenable to follow-up studies due to the brightness of the parent stars, and the favourable planet-star flux ratio. MEarth is such a transit survey of $\sim 2000$ nearby M dwarfs. Since the targets are distributed over the entire (Northern) sky, it is necessary to observe them individually, which will be done by using 8 independent $0.4 \mathrm{~m}$ robotic telescopes, two of which have been in operation since December 2007 at the Fred Lawrence Whipple Observatory (FLWO) located on Mount Hopkins, Arizona. We discuss the survey design and hardware, and report on the current status of the survey, and preliminary results obtained from the commissioning data.
\end{abstract}

\section{Introduction}

M dwarfs are very favourable targets to search for transiting super-Earth exoplanets in the habitable zones of their parent stars (e.g. Charbonneau \& Deming 2007). This is predominantly a result of the very low luminosities of $\mathrm{M}$ dwarfs relative to solar type stars, which means that the habitable zones reside at much smaller orbital distances. For the present discussion, we assume an M5-dwarf star, with mass and radius $\sim 0.25 \mathrm{M}_{\odot}$ and $0.25 \mathrm{R}_{\odot}$ respectively, orbited by a planet of $7 \mathrm{M}_{\oplus}$ and $2 \mathrm{R}_{\oplus}$, representing a typical target of the MEarth survey. Given these parameters, the M dwarf has a luminosity $\sim 1 / 200$ that of the Sun, and consequently, a planet receiving the same stellar insolation as the Earth would lie at $0.074 \mathrm{AU}$, corresponding to an orbital period of 14.8 days. The geometric transit probability for such a planet is also raised by a factor of approximately three, from $0.5 \%$ (for the Earth-Sun system) to $1.6 \%$.

The small radii of $\mathrm{M}$ dwarfs also give rise to substantially deeper transits $(0.5 \%$ for the M5-dwarf, compared to $0.03 \%$ for a solar-type host), and the combination of a smaller stellar mass and shorter orbital period increases the radial velocity semiamplitude, to $\sim 10 \mathrm{~m} \mathrm{~s}^{-1}$ compared to $1.3 \mathrm{~m} \mathrm{~s}^{-1}$ for the Earth-Sun system.

The combination of these factors means that the detection of such planets around $\mathrm{M}$ dwarfs by the transit method is feasible from the ground, using present-day observational techniques and detectors. MEarth is such a survey using modest commercially-available equipment, based on a design study published in Nutzman \& Charbonneau (2008).

In this contribution, we summarise the results of the design study, present the novel mode of operation of the MEarth survey, and describe the implementation and current status of the hardware. 


\section{Survey design}

In order to detect a few super-Earth planets, or to place meaningful constraints on their incidence, for a geometric transit probability of $\sim 1 \%$ we must survey a few thousand $\mathrm{M}$ dwarfs. These should be bright in order to allow follow-up studies to be performed, and given the intrinsic faintness of $\mathrm{M}$ dwarfs this translates to being very nearby: for example, an $\mathrm{M} 5 \mathrm{~V}$ star has an absolute J-band magnitude of $M_{J} \simeq 9$. A reasonable limit for follow-up studies is $J \sim 12$, implying distances $<40 \mathrm{pc}$.

Such nearby stars are expected to have high proper motions. We therefore appeal to the recently-completed LSPM-North catalogue (Lépine \& Shara 2005), a survey of the entire Northern hemisphere based on photographic plates, which should be nearly complete for stars with proper motion $>0.15^{\prime \prime} / \mathrm{yr}$, containing a total of $\sim 62000$ sources. We further restrict this to a sub-sample of 4131 stars within 33 pc (Lépine 2005) to remove high proper motion sub-dwarf contaminants from the sample.

In order to select the $\mathrm{M}$ dwarfs from the remaining population of (predominantly) main sequence dwarfs, we apply colour cuts using combinations of the $V$-band magnitudes from Lépine \& Shara (2005), and 2MASS $J, H$ and $K_{S}$ magnitudes, which are available for every star in our sample from the 2MASS all-sky data release (Skrutskie et al. 2006). The resulting "culled" sample consists of 3300 probable M dwarfs.

These targets are spread in a relatively uniform fashion over the entire celestial Northern hemisphere. We have therefore opted for a strategy of observing them individually. This mode of operation brings a number of benefits. In particular, the requirement on the field-of-view of the detector is substantially relaxed, since it need only be large enough to obtain sufficient comparison stars to perform differential ensemble photometry. It is also possible to tailor the parameters of each observation to the individual target $\mathrm{M}$ dwarf, for example varying the exposure time to achieve the required signal to noise to detect planets of a given size, thereby saving observing time.

Nutzman \& Charbonneau (2008) found that the most favourable targets for such a transit survey are, in fact, the smallest stars: although these are intrinsically fainter, the reduced count rates are compensated by having deeper transits, and their faintness increases the number of suitable comparison stars available for a given field-of-view. It is important to recall that for small field-of-view observations of single targets, the noise in the comparison light curve can become an important, or even dominant, contributor to the total noise budget. We therefore further choose to concentrate on the smallest stars, choosing 1976 with estimated radii $<0.33 \mathrm{R}_{\odot}$ (corresponding to a spectral type of $\sim \mathrm{M} 3$ ).

Since the emission from $\mathrm{M}$ dwarfs peaks in the near-IR, the number of detected stellar photons would be maximised by observing in this spectral region. Unfortunately, near-IR detectors remain prohibitively expensive, so MEarth uses conventional CCDs. Nutzman \& Charbonneau (2008) found that the optimal passband for observing the (extremely red) M-dwarf target stars was a filter cutting on longward of $\sim 700 \mathrm{~nm}$, and limited on the red end by the tail of the CCD quantum efficiency curve. The $\mathrm{H} \alpha$ line was deliberately omitted from the bandpass due to its potential variability in very active stars, which is obviously an undesirable feature for precision photometry. This passband approximates the sum of transmission of the Sloan $i$ and $z$ filters (Fukugita et al. 1996), and has been termed an " $i+z$ " filter. In reality, this is formed using a single piece of Schott RG715 glass, and is therefore economical and straightforward to manufacture, and the final bandpass using an e2v CCD42-40 is very similar to the conventional Cousins $I$ filter.

Given these parameters, we can calculate the telescope aperture and field-of-view required to survey our target stars. This was done assuming a standard noise model, including contributions from Poisson noise in the stellar counts and sky background, 
dark current, readout noise, and atmospheric scintillation using the formulation of Young (1967). Figure 1 indicates that a $40 \mathrm{~cm}$ aperture telescope with a $25^{\prime} \times 25^{\prime}$ field-of-view is sufficient for the vast majority of the $\mathrm{M}$ dwarfs with $R<0.33 \mathrm{R}_{\odot}$.

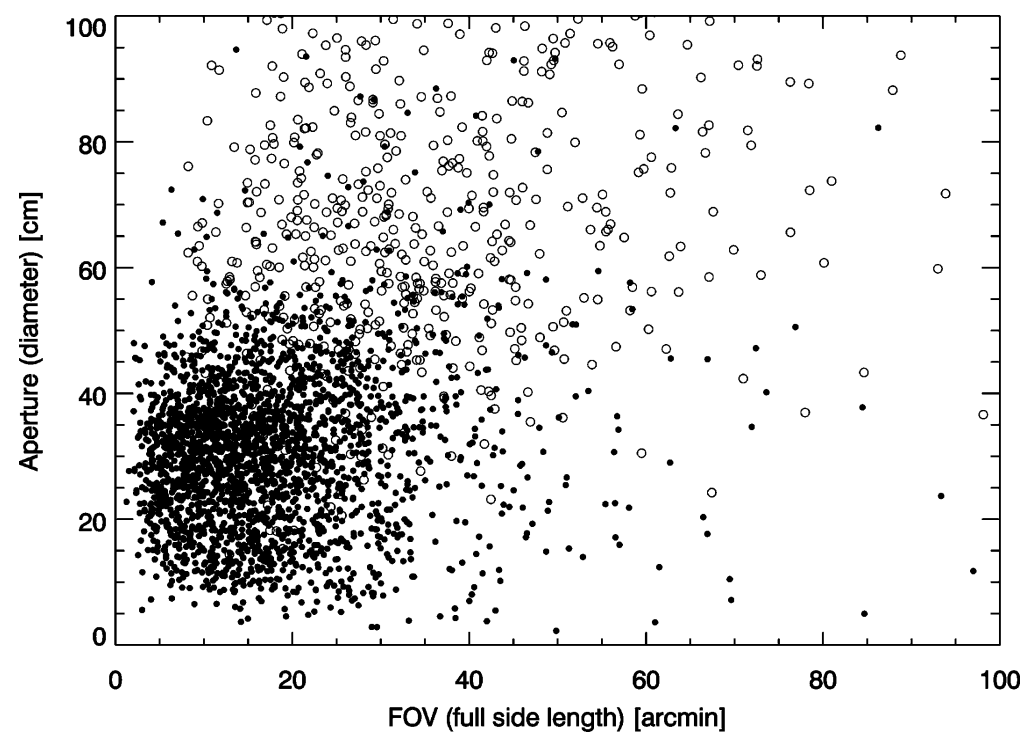

Figure 1. Necessary aperture vs. necessary field of view for LSPM M dwarfs. Stars with radius $>0.33 R_{\odot}$ are represented by open circles, while stars with radius $<0.33 R_{\odot}$ are represented by filled circles. In order to determine the required telescope aperture, we demanded the photometric precision necessary to achieve a $3 \sigma$ detection of a transiting $2 \mathrm{R}_{\oplus}$ planet in a 150 s integration through the $i+z$ filter. The range of the $\mathrm{x}$ and y axes match that of the stars with radii $<0.33 \mathrm{R}_{\odot}$, while $30 \%$ of the $\mathrm{M}$ dwarfs with radii $>0.33 \mathrm{R}_{\odot}$ require more than a $100 \mathrm{~cm}$ aperture, and thus fall above the plot limits.

We have opted for eight such telescopes, which leads to an expected 2 yr duration to complete the survey of the habitable zones of these $1976 \mathrm{M}$ dwarfs. The design study indicates that a yield of 2.6 habitable zone super-Earths would be predicted if the true occurrence of these planets was $10 \%$ around our targets, with larger and closer-in planets being easier to detect. A null result would limit the occurrence of $>2 \mathrm{R}_{\oplus}$ super-Earth planets in the habitable zones of late-M dwarfs to be $<17 \%$ at the $99 \%$ confidence level, a result that again becomes a stronger limit for closer-in planets.

\section{Mode of operation}

In order to improve the survey efficiency, and in particular, to increase the number of $\mathrm{M}$ dwarfs that can be monitored at once by each telescope, we have adopted a novel detection strategy (illustrated in Figure 2). Routine observations will be carried out at extremely low cadence, with $\sim 2$ visits to each target per transit timescale (we assume a mid-latitude transit, which leads to a duration 0.866 times that of an equatorial transit). The cadence is further limited to not being less than once every half-hour to assure a reasonable number of observations of each target per night, and to catch shorter-period transits due to planets interior to the habitable zone. We intend to detect transits while they are still in progress via real-time analysis of the images as they are taken. This information can then be used to immediately direct follow-up resources (e.g. other MEarth telescopes) 
to confirm or reject the hypothesis that there is a transit in progress. In practice, this will probably proceed by immediately obtaining several more data-points, to combat the effects of noise and light curve systematics, and then following the remainder of the event, if it turns out to be real, at high-cadence to obtain a well-sampled transit egress. By continuously re-evaluating the probability of there being a transit in-progress upon obtaining additional data-points, we can quickly reject false positives without any major effect on the remainder of the targets. We can therefore cope with a relatively high false alarm rate.

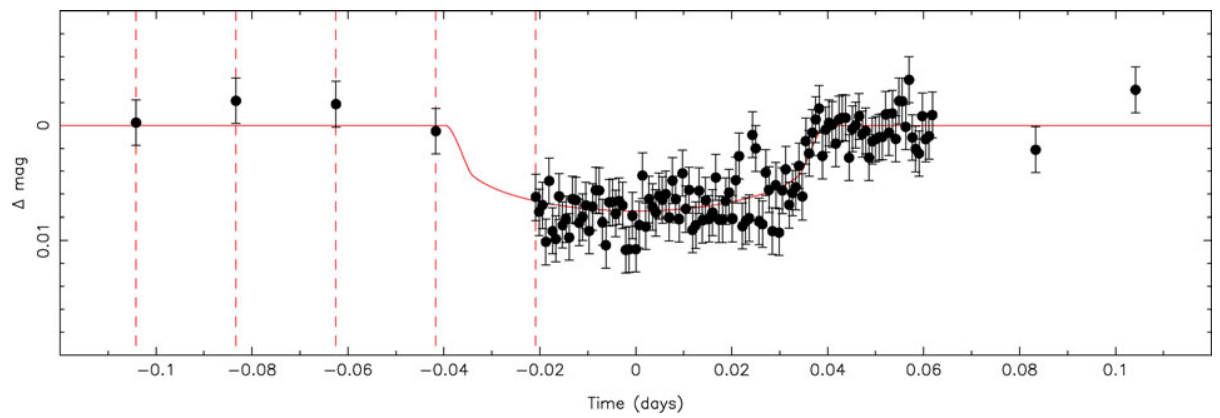

Figure 2. Illustration of a possible sampling strategy for transit detection with MEarth. The solid line shows a transit model. The dashed vertical lines indicate the sample times during the normal (low-cadence) operation up to the first point which deviates by $>1 \sigma$ in the downward direction. Immediate follow-up is triggered at this point, confirming the downward deviation. Sampling continues at high-cadence until after egress, at which point the sampling returns to normal until another significant downward light curve deviation is detected.

\section{Follow-up and false positives}

As with any transit survey, in order to confirm the planetary nature of any detections, radial velocity follow-up observations will be required. This should be possible with existing instrumentation, with several groups demonstrating the required radial velocity precision on $\mathrm{M}$ dwarfs using conventional instruments in relatively blue passbands. Ideally, in order to take advantage of the greater continuum flux of $\mathrm{M}$ dwarfs in the far red and infrared, radial velocities would be derived from lines in these spectral regions. Unfortunately the conventional Iodine cell technique is not usable in the far-red, and the HARPS (and by extension, HARPS-NEF) spectrographs cannot be used beyond $\sim 690 \mathrm{~nm}$. The new TRES spectrograph on the Tillinghast $1.5 \mathrm{~m}$ telescope at FLWO appears promising in this respect, since it can operate around the atmospheric window close to $900 \mathrm{~nm}$, as do several IR radial velocity methods currently under development, for example Blake et al. $(2007,2008)$ and the T-EDI project (Lloyd et al., this volume).

It should be noted that the target selection employed for MEarth largely eliminates the most common sources of astrophysical false positives that plague conventional wide-field transit surveys. In particular, the selection by proper motion eliminates giant host stars from the sample, and hierarchical triple systems are extremely unlikely due to the very late spectral types that they must have in order to be included in the MEarth sample (the brightest star in these would be a mid-M dwarf due to the colour selection we apply). Triples may also be resolvable with high-resolution imaging due to their close proximity to the Earth. Finally, blends with background binaries are substantially reduced, not least because our pixel scale of $0.75^{\prime \prime} /$ pix is $\sim 1 / 20$ that of a typical wide-field transit survey, and the high proper motions of our targets mean that previous epochs of imaging 
can be used to resolve many of these systems, by looking for the background source at the present position of the $\mathrm{M}$ dwarf.

\section{Status}

At the time of writing, MEarth has 5 telescopes in operation, with two operating routinely since January 2008, and the final three since June 2008. We anticipate having the remaining three telescopes operational by September 2008. All of the telescopes are housed in the same building. We note that the use of multiple sites at different longitudes would be beneficial to improve phase coverage, but would be much more expensive, since we were able to re-use an existing building and much of the associated support infrastructure at FLWO, affording a very substantial cost saving.

Data are presently reduced in real time, although the real-time analysis software is not yet in place, using a modified version of the pipeline from the Monitor project, described in Irwin et al. (2007). We have already achieved photometry at the $\sim 0.6 \%$ level on M-dwarf targets over 90 nights, and are currently investigating methods to further reduce the level of systematics present in the data. This has been sufficient to detect low-amplitude (few percent peak-to-peak) rotational modulations in several of our targets for which sufficient observations are available.

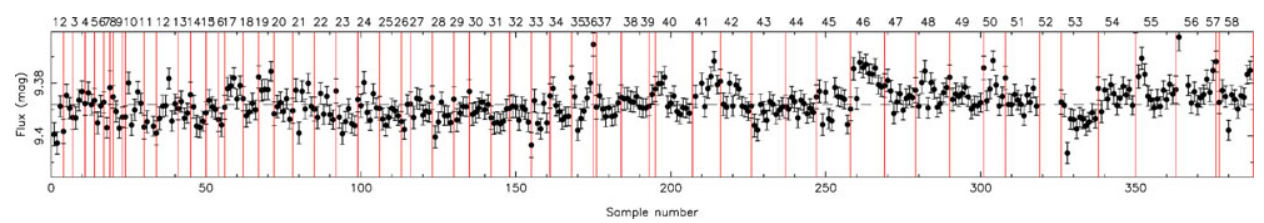

Figure 3. Example of an M-dwarf light curve from MEarth data taken during the first season of operations, plotted in $I$ magnitude as a function of sample number (numbering from 1 for the first data point). Vertical solid lines denote boundaries between different nights of observations, which are numbered at the top of the diagram. Data were taken on 58 separate nights for this object. The estimated spectral type of the star is M4V, with an I-band magnitude of 29.4 .

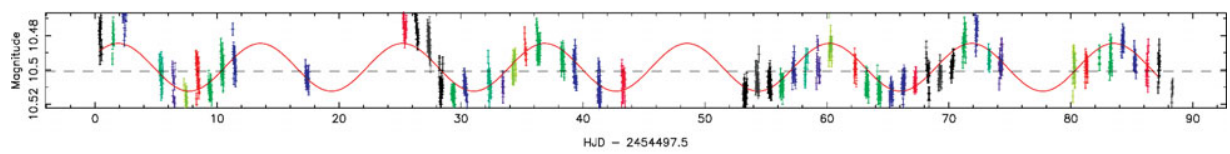

period 11.661

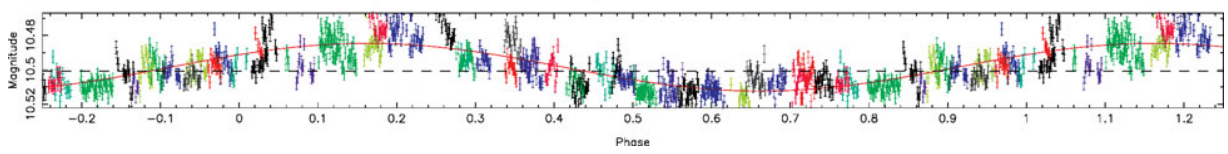

Figure 4. Example of an M-dwarf light curve showing periodic photometric variations, presumably due to rotation. The upper panel shows the light curve plotted as a function of heliocentric Julian day number, and the lower panel shows the curve folded on the best-fitting rotation period of 11.661 days, plotted versus orbital phase, where 1 unit represents a single rotational period. The estimated spectral type of the star is $\mathrm{M} 4.5 \mathrm{~V}$, with an I-band magnitude of $\sim 10.5$.

A new M-dwarf eclipsing binary system was also discovered during the first few weeks of routine MEarth observations. The system is a near equal mass pair, with estimated component masses $\sim 0.3 \mathrm{M}_{\odot}$ from photometry, and an extremely short orbital period of 0.77 days. The light curve of this object obtained using one MEarth telescope is shown in Figure 5. 


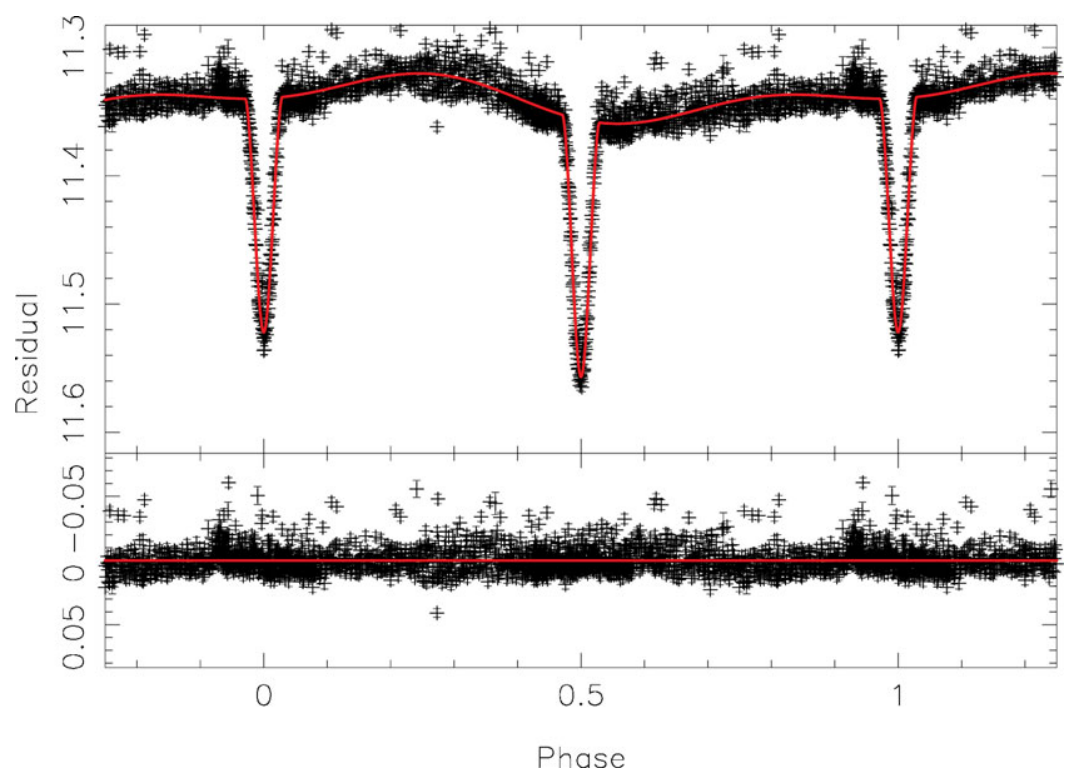

Figure 5. Phase-folded light curve of the M-dwarf eclipsing binary discovered in MEarth data. The upper panel shows the $I$-band magnitude, over-plotted with an eclipsing binary model (solid line) generated using a modified version of JKTEBOP (Southworth et al. 2004a,b; Popper \& Etzel 1981; Etzel 1981) including out-of-eclipse modulations (presumably due to starspots) synchronised with the binary orbital period of 0.77 days. The lower panel shows the residuals (data - model) of the fit. Note the differing scales on the vertical axes in the two panels.

Such eclipsing binaries are of vital importance for constraining the mass-radius relation on the main sequence, and hence theoretical models of low-mass stellar evolution, which are remarkably poorly constrained even at old, main sequence ages for stars below $1 \mathrm{M}_{\odot}$. Indeed, many of the observed and well-characterised systems show substantial discrepancies with the predictions of the theoretical models (e.g. Ribas 2006). These uncertainties have a direct impact on the reliability of stellar parameter estimates, and hence on planet properties inferred from analysis of transiting systems. Indeed, as detailed elsewhere in this volume, many of the transiting planet observations have reached sufficient quality that this uncertainty in the parameters of the host star is the limiting factor in our knowledge of the properties of the planets. Such M-dwarf eclipsing binaries are straightforward to detect from MEarth data, and it should be possible to characterise them extremely well due to their brightness, allowing model-independent mass and radius estimates with uncertainties of only a few percent to be made (e.g. Andersen 1991).

\section{Acknowledgments}

It is a pleasure to acknowledge the assistance of the staff at the Fred Lawrence Whipple Observatory in Arizona: Karen Erdman-Myres, Grace Alegria, Rodger Harris, Dave Martina, Dennis Jankovsky, Tom Welsh, Wayne Peters, Ted Groner, Bob Hutchins, Perry Berlind, Mike Calkins, and Gil Esquerdo; and the Harvard-Smithsonian Center for Astrophysics: Irene Coyle, Andrea Moore, Maureen Connors, Jean Collins, Sara Yorke, and Leslie Feldman. We acknowledge funding from the David and Lucile Packard Fellowship for Science and Engineering, Harvard University, and the U.S. National Science Foundation. This work has made use of data products from the Two Micron All Sky Survey, which is a joint project of the University of Massachusetts and the Infrared 
Processing and Analysis Center / California Institute of Technology, funded by the National Aeronautics and Space Administration and the National Science Foundation.

\section{References}

Andersen, J. $1991 A \& A R v, 3,91$

Blake, C. H., Charbonneau, D., White, R. J., Marley, M. S., \& Saumon, D. 2007 ApJ, 666, 1198

Blake, C. H., Charbonneau, D., White, R. J., Torres, G., Marley, M. S., \& Saumon, D. 2008 ApJ, 678, 125

Charbonneau, D. \& Deming, D. 2007, The Dynamics-Based Approach to Studying Terrestrial Exoplanets, submitted to the Exoplanet Task Force (AAAC), 2 April 2007, arXiv:0706.1047

Etzel, P. B. 1981, in Carling E. B., Kopal Z., eds, Photometric and Spectroscopic Binary Systems, NATO ASI Ser. C., 69. Kluwer, Dordrecht, p. 111

Fukugita, M., Ichikawa, T., Gunn, J. E., Doi, M., Shimasaku, K., \& Schneider, D. P. 1996, AJ, 111,1748

Irwin, J., Irwin, M., Aigrain, S., Hodgkin, S., Hebb, L., \& Moraux, E. 2007, MNRAS, 375, 1449

Lépine, S. 2008, $A J, 130,1680$

Lépine, S. \& Shara, P. 2008, AJ, 129, 1483

Nutzman, P. \& Charbonneau, D. 2008, PASP, 120, 317

Popper, D. M. \& Etzel, P. B. 1981, AJ, 86, 102

Ribas, I. 2006 ApESSS, 304, 89

Southworth, J., Maxted, P. F. L., \& Smalley, B. 2004a, MNRAS, 351, 1277

Southworth, J., Zucker, S., Maxted, P. F. L., \& Smalley, B. 2004b, MNRAS, 355, 986

Skrutskie, M. F., et al. 2006, AJ, 131, 1163

Young, A. T. 1967, AJ, 72, 747 